\title{
Pancreaticoduodenectomy with Mesocaval Shunt for Locally Advanced Pancreatic Adenocarcinoma
}

\author{
Eve Simoneau, MD, $\mathrm{PhD}^{1}$, Claire Goumard, $\mathrm{MD}^{2}$, Jeffrey E. Lee, $\mathrm{MD}^{\mathbf{1}}$, Jean-Nicolas Vauthey, $\mathrm{MD}^{\mathbf{1}}$, \\ Thomas A. Aloia, MD ${ }^{1}$, Yun Shin Chun, MD ${ }^{1}$, Michael P. Kim, MD ${ }^{1}$, Matthew H. G. Katz, MD ${ }^{1}$, \\ and Ching-Wei D. Tzeng, MD ${ }^{1}$
}

${ }^{1}$ Department of Surgical Oncology, The University of Texas MD Anderson Cancer Center, Houston, TX; ${ }^{2}$ Department of Hepatobiliary Surgery and Liver Transplantation, La Pitié Salpetrière Hospital, Université Pierre et Marie Curie (UPMC), Assistance Publique-Hôpitaux de Paris, Paris, France

\begin{abstract}
Introduction. Patients with locally advanced pancreatic cancer (LAPC) represent a challenging group to treat, given the involvement of major vascular structures. In selected patients with favorable biology, temporary mesocaval shunt can facilitate the resection and allow for a safer procedure with enhanced exposure to the superior mesenteric vessels.

Methods. We present a video of a pancreaticoduodenectomy (PD) with temporary mesocaval shunt with left internal jugular (LIJ) vein conduit.

Results. A 65-year-old woman presented with LAPC in the uncinate, causing total occlusion of the superior mesenteric vein (SMV) and encasement of the first jejunal artery. After neoadjuvant therapy and evidence of disease stability, a decision was made to perform a PD with a temporary mesocaval shunt to divert mesenteric flow to reduce blood loss and prevent bowel ischemia. During the
\end{abstract}

Video is reprinted with modification from HPB Journal (HepatoPactreato-Biliary), vol 20/supplement 1, Simoneau E, Goumard C, Lee JE, et al., Pancreaticoduodenectomy with mesocaval shunt for locally advanced pancreatic adenocarcinoma, page S111, copyright 2018, with permission from Elsevier.

Electronic supplementary material The online version of this article (https://doi.org/10.1245/s10434-018-07093-x) contains supplementary material, which is available to authorized users.

(C) Society of Surgical Oncology 2018

First Received: 30 September 2018;

Published Online: 11 December 2018

C.-W. D. Tzeng, MD

e-mail: CDTzeng@mdanderson.org procedure, the main mesenteric collateral (ileocolic vein) was divided to create the shunt to the inferior vena cava (IVC) with LIJ interposition. The remaining mesenteric tributaries involved by the tumor were divided. The uncinate dissection was performed using a superior mesenteric artery-first approach. Once the resection was completed, the shunt was stapled from the IVC and the graft transposed to the upper SMV. Standard reconstruction was performed. Total operative time was $536 \mathrm{~min}$, and estimated blood loss was $250 \mathrm{cc}$ without transfusions. No perioperative complications occurred.

Conclusion. In selected patients with LAPC, PD with temporary mesocaval shunt can facilitate resection and venous reconstruction in patients with complete portal vein/SMV occlusion.

DISCLOSURE Eve Simoneau, Claire Goumard, Jeffrey E. Lee, Jean-Nicolas Vauthey, Thomas Aloia, Yun-Shin Chun, Michael P. Kim, Matthew Katz, and Ching-Wei Tzeng have not declared any conflicts of interest. 